This is the final peer-reviewed accepted manuscript of:

R Longo et al., Towards breast tomography with synchrotron radiation at Elettra: first images, 2016 Phys. Med. Biol. 611634.

The final published version is available at: https://doi.org/10.1088/0031$\underline{9155 / 61 / 4 / 1634}$

Rights / License:

The terms and conditions for the reuse of this version of the manuscript are specified in the publishing policy. For all terms of use and more information see the publisher's website. 


\section{Towards breast tomography with synchrotron radiation at Elettra: first images}

\section{R Longo ${ }^{1,2}$, F Arfelli ${ }^{1,2}$, R Bellazzini ${ }^{3,4}$, U Bottigli ${ }^{5}$, A Brez ${ }^{3,4}$, F Brun ${ }^{2,6}$, A Brunetti ${ }^{7}$, P Delogu ${ }^{4,8}$, F Di Lillo ${ }^{9}$, D Dreossi ${ }^{10}$, V Fanti $^{11}, C_{\text {Fedon }}{ }^{1,2}$, B Golosio $^{7}$, N Lanconelli ${ }^{12}$, G Mettivier ${ }^{9}$, M Minuti ${ }^{3,4}$, P Oliva ${ }^{7}$, M Pinchera $^{3,4}$, L Rigon ${ }^{1,2}$, P Russo ${ }^{9}$, A Sarno ${ }^{9}$, G Spandre ${ }^{3,4}$, G Tromba ${ }^{10}$ and F Zanconati ${ }^{13}$}

${ }^{1}$ Department of Physics, University of Trieste, Trieste, Italy

${ }^{2}$ INFN Sezione di Trieste, Trieste, Italy

${ }^{3}$ PiXirad Imaging Counters srl, Pisa, Italy

${ }^{4}$ INFN Sezione di Pisa, Pisa, Italy

${ }^{5}$ Department of Physics, University of Siena, Siena, Italy

${ }^{6}$ Department of Engineering and Architecture, University of Trieste, Trieste, Italy

${ }^{7}$ University of Sassari \& INFN Sezione di Cagliari, Sassari, Italy

${ }^{8}$ Department of Physics, University of Pisa, Pisa, Italy

${ }^{9}$ Department of Physics, University of Naples Federico II \& INFN Sezione di Napoli, Naples, Italy

${ }^{10}$ Elettra-Sincrotrone Trieste S.C.p.A, Basovizza, Trieste, Italy

${ }^{11}$ Department of Physics, University of Cagliari \& INFN Sezione di Cagliari, Cagliari, Italy

${ }^{12}$ Department of Physics and Astronomy, University of Bologna \& INFN Sezione di Bologna, Bologna, Italy

${ }^{13}$ Department of Medical Science, Pathology Unit, Cattinara Hospital, University of Trieste, Trieste, Italy

E-mail: renata.longo@ts.infn.it

\footnotetext{
Abstract

The aim of the SYRMA-CT collaboration is to set-up the first clinical trial of phase-contrast breast CT with synchrotron radiation (SR). In order to combine high image quality and low delivered dose a number of innovative elements are merged: a CdTe single photon counting detector, state-of-the-art
} 
$\mathrm{CT}$ reconstruction and phase retrieval algorithms. To facilitate an accurate exam optimization, a Monte Carlo model was developed for dose calculation using GEANT4. In this study, high isotropic spatial resolution $(120 \mu \mathrm{m})^{3} \mathrm{CT}$ scans of objects with dimensions and attenuation similar to human breast were acquired, delivering mean glandular doses in the range of those delivered in clinical breast CT (5 - $25 \mathrm{mGy})$. Due to the spatial coherence of the SR beam and the long distance between sample and detector, the images contain not only absorption but also phase information from the samples. The application of a phase-retrieval procedure increases the contrast-to-noise ratio of the tomographic images, while the contrast remains almost constant. After applying Simultaneous Algebraic Reconstruction Technique (SART) to low-dose phase-retrieved data sets (about $5 \mathrm{mGy}$ ), obtained by reducing the number of projections, the spatial resolution was equal to the one obtained with filtered back projection with a four times higher dose, while the contrast-to-noise ratio was reduced by $30 \%$. These first results indicate the feasibility of clinical breast CT with SR.

\section{Introduction}

In x-ray breast imaging the challenge is to match high image quality and acceptable delivered dose. In the last decades an increasing effort in the transition from planar imaging (digital mammography) to 3D imaging can be noticed in the clinical practice. The development of new digital detectors and powerful reconstruction algorithms allows the realization of 3D mammographic systems, such as tomosynthesis and tomographic scanners. Breast tomosynthesis (Sechopoulos 2013a,b) is widely used in clinical exams and very good results have been published even if the images show intermediate quality between mammography and tomography in terms of voxel size and contrast- A few cone beam breast CT prototypes are in clinical use in the world (Prionas et al 2010, O'Connell et al 2014, Zhao et al 2015); the main constraint in the development of this technique is the necessary limitation of the delivered dose (Kalender et al 2012, Vedantham et al 2013, Sarno et al 2015).

At the same time, the so-called $\mathrm{x}$-ray phase-contrast $(\mathrm{PhC})$ imaging techniques have been investigated in mammography with promising results (Coan et al 2013). The first x-ray PhC mammography clinical study performed with synchrotron radiation (SR), based on the propagation-based $\mathrm{PhC}$ technique, demonstrated an improvement in the image quality and in the diagnostic performance without increasing the delivered dose compared to conventional mammography (Castelli et al 2011, Longo et al 2014). The transition of PhC mammography from SR radiation facilities to the hospitals is a research topic for a number of groups (Tanaka et al 2005, Bravin et al 2013).

The application of $\mathrm{PhC}$ techniques to tomographic imaging of breast tissues is quite promising, particularly when the phase and absorption signals are studied separately (Brun et al 2014, Grandl et al 2014). In conventional tomography the images represent maps of the x-ray attenuation coefficients of the sample. In propagation-based $\mathrm{PhC}$ tomography the phase information can be decoupled from absorption when a phase retrieval algorithm is applied to the projections before tomographic reconstruction (with suitable approximations on the sample properties). This procedure increases the contrast resolution in the reconstructed images (Cloetens et al 1999, Paganin et al 2002, Chen et al 2013). The benefits of phase retrieval when using an algorithm based on the homogeneous transport of intensity equation (Paganin et al 2002) are well acknowledged. In general, the application of phase retrieval results in reduced noise while 
preserving the sharpness of the edges when used with a proper parameter quantifying the relationship between the imaginary part $(\beta)$ and the decrement from unity $(\delta)$ of the complex refraction index $n=1-\delta+$ i $\beta$ of the object (e.g. Beltran et al 2011; Nesterets and Gureyev 2014). The application of this approach has already proved to be beneficial also for breast tomography (e.g. Pacilè et al 2015; Nesterets et al 2016).

In x-ray imaging, high efficiency, direct conversion and photon counting detectors show great advantages with respect to detectors based on indirect detection and charge integration. The most remarkable advantage of photon counting is the possibility to obtain a Poisson-dominated signal-to-noise ratio, cutting out the electronic noise. The intrinsic limit in photon counting is the non-linearity, especially at high photon fluxes, due to dead time in the electronics. Direct detection by thick layers of high- $Z$ semiconductors produces high detection efficiency and minimizes the loss of spatial resolution due to light scattering in scintillator-based systems. At equal sampling steps, direct detection systems may show, with respect to scintillator-based ones, better spatial resolution (Taguchi and Iwanczyk 2013). The first photon counting detectors developed for SR mammography were based on silicon microstrip sensors in edge-on geometry (Arfelli et al 1997, Arfelli et al 1998, Longo et al 2003). When the silicon microstrip detectors were used in a commercial mammography unit, a significant reduction in the delivered dose was recorded, mainly in the exam of thick breast (Åslund $e t$ al 2007, Weigel et al 2014). The dose reduction is based on the high Detective Quantum Efficiency (DQE) of the system and on the higher energy spectra that can be used with the digital detector compared with the screen-film systems (Quai et al 2012). A new generation of photon-counting direct-conversion detectors are available, based on cadmium telluride (CdTe)(e.g. Chmeissani et al 2004). In contrast with Si microstrip detectors, which are 1D sensors (Arfelli et al 1997, Åslund et al 2007), the CdTe sensors are 2D pixel detectors available with different tile-up capability (Taguchi and Iwanczyk 2013, Bellazzini et al 2013).

While high detector DQE can be instrumental in order to reduce the delivered dose in CT, it is not the only characteristic of the system that plays a crucial role: to this purpose, a number of techniques have been developed in both data acquisition protocol and in reconstruction algorithms. The filtered back projection (FBP) algorithm is one of the most popular algorithms for CT reconstruction; however, it is well known that it produces significant image artefacts when only a reduced number of projections are acquired. In fact, when the number of projections is significantly lower than the minimum number prescribed by the Nyquist radial sampling criteria, the tomographic reconstruction represents an ill-posed problem, and conventional reconstruction algorithms do not yield adequate results (Kak and Slaney 2001, Buzug 2008). Several approaches have been proposed to deal with this problem, including SIRT (Simultaneous Iterative Reconstruction Technique), SART (Simultaneous Algebraic Reconstruction Technique) (Gordon et al 1970), CGLS (Conjugate Gradient Least Square) (van der Sluis and van der Horst 1990), MLEM (Maximum Likelihood Expectation Maximization) (Dempster et al 1977), MR-FBP (Minimum Residual FBP) (Pelt and Batenburg 2014) and morphing techniques (Brunetti and Golosio 2001). Some of them have been applied to breast CT data, including iterative reconstruction algorithms with appropriate filters (Makeev and Glick 2013, Bian et al 2014) and the so-called Equally Slope Tomography (EST), which combines an acquisition 
with a non-equally spaced angular sampling of projections with a Fourier reconstruction algorithm (Miao et al 2005, Zhao et al 2012).

The aim of the SYRMA-CT (SYnchrotron Radiation MAmmography-Computed Tomography) collaboration is to set-up the first clinical trial of PhC breast CT with SR at the SYRMEP (SYnchrotron Radiation for MEdical Physics) beamline of Elettra, the synchrotron radiation laboratory in Trieste (Italy). Pursuing both high image quality and low delivered dose, a number of innovative elements will be merged: a novel CdTe single photon counting detector (PIXIRAD-8, Bellazzini et al 2013), state-of-the-art CT reconstruction techniques, phase retrieval algorithm. In order to perform an accurate exam optimization, a Monte Carlo model has been developed for dose calculation using the GEANT4 simulation toolkit (Agostinelli et al 2003). In this paper we present the key elements of the project and discuss the first images of a mammographic test object and of two breast tissue specimens.

\section{Material and Methods}

\subsection{The beamline and acquisition set-up}

The images were acquired at the SYRMEP (SYnchrotron Radiation for MEdical Physics) beamline at Elettra. The radiation source is one of the storage ring bending magnets; the beam is monochromatized by means of a $\mathrm{Si}(111)$ double-crystal monochromator, which provides a beam energy in the range $8.5 \mathrm{keV}-38$ $\mathrm{keV}$, with a resolution of about $0.2 \%$. Several tungsten slit systems are used to define the beam shape. The beam maximum cross section in the radiology hutch is $220 \mathrm{~mm}$ (horizontal) $\times 5 \mathrm{~mm}$ (vertical, Gaussian shape, FWHM) (Abrami et al 2005). A dosimetric system, developed for clinical planar mammography (Castelli et al 2011), was upgraded for clinical tomography. The SYRMEP patient support was designed in order to allow mammography, by scanning the compressed breast in the vertical direction, and breast CT acquisition, by the rotation of the patient with the breast to be imaged hanging in a pendant geometry outside of an ergonomically designed aperture at the rotation centre (Pani et al 2004). The phase sensitive technique exploited in the clinical studies at the SYRMEP beamline is the so called propagation-based phase-contrast and it is based on the high coherence of the SR beam and on the long propagation distance between patient and detector $(\sim 2 \mathrm{~m})$; moreover to detect the edge-enhancement effect associated to this $\mathrm{PhC}$ technique the spatial resolution of the detector has to be adequately high (Arfelli et al 1998), since no optical elements are

present in the beam path and the transverse diffraction pattern in the near field has to be very finely sampled in space.

The preliminary images reported in this paper were acquired in a simplified set-up using a rotation stage at the breast position in the radiology hutch. The beam energy was $38 \mathrm{keV}$ : a good compromise between optimal energy for the CT of breast with SR and efficient response of the CdTe detector (Mittone et al 2014), within the energy range of the SYRMEP beamline. The projections were collected over 180 degrees, thanks 
to the negligible horizontal divergence of the beam $(7 \mathrm{mrad})$, which permits acquisition in parallel beam geometry. The vertical beam dimension was $3 \mathrm{~mm}$.

\subsection{Dose evaluation}

Due to the laminar shape of the SR beam, acquisition of a breast volume requires multiple rotations of the patient. The reduced breast volume under investigation will be selected during the exam initialization and it will be covered by collecting several $\mathrm{CT}$ axial scans at different adjacent vertical positions, in a rotate/translate scan geometry. Taking into account the available beam height and according to the diagnostic requirements, the number of slices will be defined in each exam: it is expected to be in the range 10-20 (3-6 cm axial length).

In a mammography exam, the calculation of the mean glandular dose (MGD) assumes that the whole breast is exposed to the x-rays (Hammerstein et al 1979) but, in the case of this work, only a partial breast irradiation will occur: an extension of the mean glandular dose metric has been defined for the present research program (Mettivier et al 2015). The glandular absorbed dose in the irradiated volume $\left(\mathrm{MGD}_{\mathrm{v}}\right)$ is defined as the average dose delivered to the glandular breast component in the irradiated CT slice. The $\mathrm{MGD}_{\mathrm{v}}$ is calculated by multiplying the value of the air kerma at the breast position (scanner isocentre) with a conversion factor (function of breast size and glandular fraction) obtained from a Monte Carlo simulation developed for this study (Mettivier et al 2015) based on a GEANT4 code optimized for breast dosimetry (Fedon et al 2015).

The value of the air kerma was provided by the dosimetric system developed for the clinical mammography trial (Castelli et al 2011): two ionization chambers are placed $3 \mathrm{~m}$ upstream from the breast in the beam preparation hutch, just before the radiological room. These chambers have been calibrated in the range 18-38 keV by means of a Radcal ionization chamber (mod. 10X6-3CT, Radcal, Monrovia, CA, USA) connected with the dosimeter Accu-Pro (Radcal), placed at the scanner isocenter.

\subsection{PIXIRAD and data pre-processing}

In this project, a high-efficiency photon-counting direct-conversion x-ray imaging detector is used. The basic block has a hybrid architecture in which the sensor and readout electronics, based on an application-specific integrated circuit (ASIC), are manufactured and processed separately and then coupled with the flip-chip bump-bonding technique. The sensor (ACRORAD Co., Ltd.) is a Schottky type diode array with electron collection on the pixels, made of crystalline CdTe substrate with an area of $30.96 \mathrm{~mm} \times 24.98 \mathrm{~mm}$ and thickness $0.65 \mathrm{~mm}$. The pixels are arranged on a honeycomb matrix with a pitch of $60 \mu \mathrm{m}$. The ASIC is a CMOS VLSI chip with an active area of $30.7 \mathrm{~mm} \times 24.8 \mathrm{~mm}$, organized on a honeycomb matrix of $512 \times$ 476 pixels (Bellazzini et al 2013). Each pixel incorporates a hexagonal electrode (top metal layer) connected to a charge amplifier, which feeds two discriminators, and two 15-bit counters. Each counter can be written 
while the other is read. Using this feature it is possible to realize the so-called Dead Time Free modality where no events are lost over the acquisition time. To reduce unavoidable pixel-to-pixel DC offset variations on the discrimination level, an auto-zero or self-calibration circuit is implemented in each pixel. In this way a single global threshold per discriminator can be applied to the entire matrix (Bellazzini et al 2013). The detector is produced by PIXIRAD Imaging counters s.r.l. (Pisa, Italy), a spin-off company of INFN (the Italian National Institute for Nuclear Physics).

The multi-block module used in this project is an 8-unit system (PIXIRAD-8) reaching a global active area of $250 \mathrm{~mm} \times 25 \mathrm{~mm}$, therefore raw images have $4608 \times 476$ pixels (figure 1 ). Because of the modular structure of the device, a dead space ( $60 \mu \mathrm{m}$ wide) between adjacent blocks is present. The system has been previously tested at the SYRMEP beamline in order to establish the energy resolution performance (Vincenzi et al 2015) and to verify the linearity at the photon rates that are expected during breast CT scans. The system has a linear response up to $2 \times 10^{5}$ photons per pixel per second.

All acquisitions were taken with an exposure time of $50 \mathrm{~ms}$ per projection and setting up a discriminator threshold value equivalent to half the energy of photons (i.e. $19 \mathrm{keV}$ ). This allows minimizing chargesharing effects (double counts, loss of spatial resolution) avoiding loss of counts (Lopez et al 2014). The maximum flux on the detector during the data acquisition was $610^{4}$ photons per pixel per second, far below the non-linear region.

Projection images were pre-processed in the following way:

a) Flat-field equalisation, to correct the residual pixel-to-pixel offset differences;

b) Resampling, to change from honeycomb to square sampling. This procedure, based on linear interpolation, produces raw images with $30 \mu \mathrm{m}$ pixel size;

c) Seaming, to bind adjacent blocks and close the dead space. This operation is also based on linear interpolation and involves three columns of pixels next to the edge of the block.

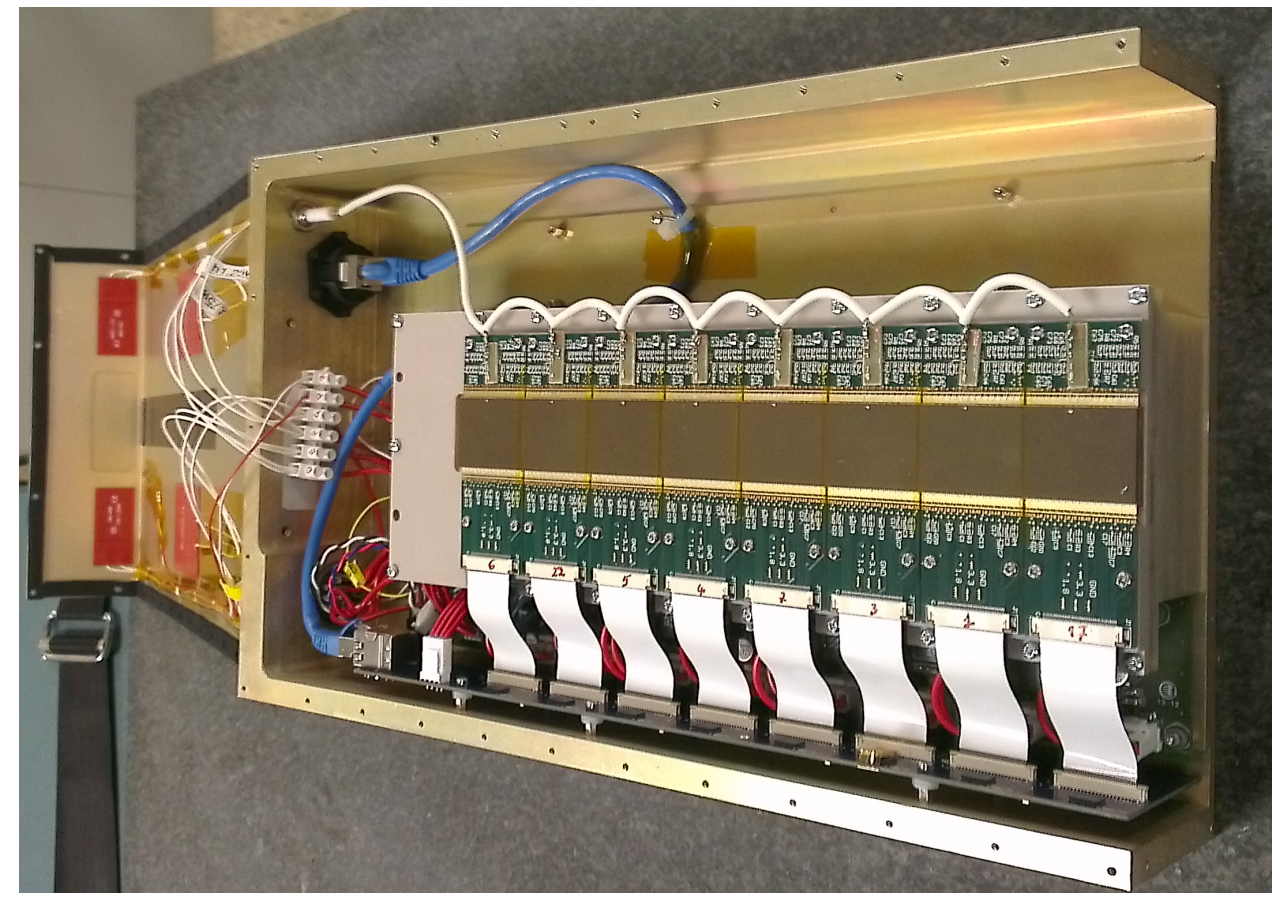


Figure 1. The PIXIRAD-8 unit: a 2 Megapixels, 4 Megacounters CdTe detector system, read-out at 30 frames/s.

\subsection{Image reconstruction}

In this preliminary study, we used two reconstruction algorithms: a standard FBP algorithm and an iterative SART algorithm. FBP reconstruction on datasets with a relatively large number of projections was used as a gold standard to evaluate the performance of other reconstruction algorithms with reduced number of projections and phase-retrieval pre-processing.

The SART algorithm has been successfully applied to a wide range of CT applications (Camerani et al 2004, Golosio et a 2004, Cauzid et al 2007); it is relatively fast compared to other iterative algorithms, and its convergence properties have been well studied (Kak and Slaney 1988). In the SART algorithm, the iterative corrections are made angle-by-angle, i.e. for each angular view, the corrections are evaluated and applied simultaneously to all rays of the projection. One iteration is considered to be completed when all the projections have been used. We used a random ordering for the projections in order to avoid systematic errors in the reconstruction. A few iterations are sufficient for the algorithm to converge. The reconstructions presented in this work were made using five iterations. The correction was multiplied by a relaxation factor, which grows linearly from zero to a maximum in the first few projections used by the reconstruction algorithm (the first 10 in this work) and decreases linearly from this maximum to zero when the number of projections increases. The relaxation factor suppresses residual oscillations, ensuring stability and convergence of the iteration (Golosio et al 2004). A bilateral filter was combined with the iterative reconstruction in order to improve image regularization. This type of filter takes two parameters: a spatial filter parameter, $\alpha_{\mathrm{d}}$, which controls the amount of smoothing differences in coordinates, and a range filter parameter $\alpha_{\mathrm{r}}$, which accounts for smoothing differences in intensities. In this work $\alpha_{\mathrm{d}}$ is expressed in pixel units, while $\alpha_{\mathrm{r}}$ is expressed in $\mathrm{cm}^{-1}$. The latter are the same units used for the linear absorption coefficient distribution obtained by the tomographic reconstruction algorithms. The optimization of the filtering was beyond the aim of this preliminary investigation.

In order to exploit the phase information carried out by the projections, a phase retrieval filter following the Homogeneous Transport of Intensity (TIE-Hom) Algorithm (Paganin et al 2002) was applied prior to the actual reconstruction, with a fixed value of the $\delta / \beta$ ratio, namely 2508 . This value was extracted from a publicly available database (https://ts-imaging.net/Services/Simple/ICUtilXdata.aspx) for the adipose tissue at $38 \mathrm{keV}$. The projections obtained by applying the phase retrieval algorithm were normalized to the same average intensity as the projections without phase retrieval. The phase retrieval pre-processing were applied to the data sampled at $60 \mu \mathrm{m}$ pixel size, even if the image was reconstructed with $120 \mu \mathrm{m}$ pixel size, in order to maximize the effectiveness of the algorithm, which is based on edge-enhancement signals.

In the following sections, the image contrast (C) and the contrast-to-noise ratio (CNR) are evaluated according the following definition: 


$$
\begin{gathered}
C=\frac{\left|S_{1}-S_{2}\right|}{S_{2}} \\
C N R=\frac{\left|S_{1}-S_{2}\right|}{\sqrt{\frac{\sigma_{1}^{2}+\sigma_{2}^{2}}{2}}}
\end{gathered}
$$

where $S$ is the average voxel value in the selected region of interest (ROI) and $\sigma^{2}$ is the signal variance in the same region. The subscript numbers indicate the two ROI between which C and CNR were evaluated.

\subsection{Samples and acquisition parameters}

Images of a multi-slab mammographic test object (CIRS mammographic phantom BR50/50 model 014AD) were acquired and the slab including the details was aligned in the beam for the tomographic acquisition. Acquisition parameters were: air kerma rate $0.29 \mathrm{mGy} / \mathrm{s}, 720$ projections equally spaced over 180 degree, 50 ms per projection. The pixel size of the reconstructed images is $60 \mu \mathrm{m}$.

Two breast tissues (Sample 1 and Sample 2) containing cancer were studied. Both of them were fixed in formalin and sealed in a vacuum bag. The work reported in this paper was carried out following the Directive 2004/23/EC of the European Parliament and of the Council of 31 March 2004 on setting standards of quality and safety for the donation, procurement, testing, processing, preservation, storage and distribution of human tissues.

The images presented in this study were acquired in order to guide the pathologist in the localization of the lesions for the histological preparation, according to the standard procedures of the Pathology Unit of the Academic Hospital of Cattinara, Trieste University, accredited by JCI (Joint Commission International). The samples were prepared from specimens of breast lumpectomy and were derived from surgical material sent to the Pathology Unit of the University Hospital of Trieste (Italy) according to local guidelines for histological examination.

Sample 1 contains an infiltrating ductal carcinoma, about $1.5 \mathrm{~cm}$ diameter. It is $8-\mathrm{mm}$ thick and it was embedded in agar gel inside a plastic cup in order to obtain an object with dimension and attenuation comparable to that of a breast section. The diameter of the holder is about $9.4 \mathrm{~cm}$. Acquisition parameters were: air kerma rate $0.52 \mathrm{mGy} / \mathrm{s}, 1200$ projections equally spaced over 180 degrees, $50 \mathrm{~ms}$ per projection, $\mathrm{MGD}_{\mathrm{v}}$ equal to $23 \mathrm{mGy}$ evaluated with a MC conversion factor $0.73 \mathrm{mGy} / \mathrm{mGy}$.

Sample 2 contains a large $(2.5 \mathrm{~cm}$ diameter) solid papillary carcinoma (poorly differentiated, G3 grade). Sample dimensions are about $89 \mathrm{~mm} \times 36 \mathrm{~mm}, 5 \mathrm{~mm}$ thickness. Acquisition parameters were: air kerma rate $0.36 \mathrm{mGy} / \mathrm{s}, 1200$ projections equally spaced over 180 degrees, $50 \mathrm{~ms}$ per projection, $\mathrm{MGD}_{\mathrm{v}}$ equal to 17 $\mathrm{mGy}$ evaluated with a MC conversion factor $0.77 \mathrm{mGy} / \mathrm{mGy}$.

\section{Results}


A detail of the mammographic phantom is presented in figure 2: It is a glandular-fat step-wedge and it is composed by two tissue-equivalent materials, i.e. glandular and fat tissue. The reconstruction in figure $2 \mathrm{a}$ was made using the FBP algorithm without phase retrieval, while the one in figure $2 \mathrm{~b}$ was made using a Paganin filter on the tomographic projections after pre-processing and before the FBP reconstruction. Figure $2 \mathrm{c}$ and $2 \mathrm{~d}$ show the signal intensity profiles of the step-wedge in the images $2 \mathrm{a}$ and $2 \mathrm{~b}$ respectively. It can be observed that phase retrieval yields significant noise reduction. The CNR evaluated on the two tissueequivalent materials was 0.23 for the reconstruction without phase retrieval, and 1.86 for the one with phase retrieval. The full width half maximum (FWHM) of the line spread function, which was evaluated through a fit of the edge profiles with the error function (erf), was $310 \mu \mathrm{m}$ and $375 \mu \mathrm{m}$, respectively. It is important to point out that, although the spatial resolution of the reconstruction without phase retrieval is (slightly) higher, it does not allow for recognition of very small details, because of the relevant noise level.

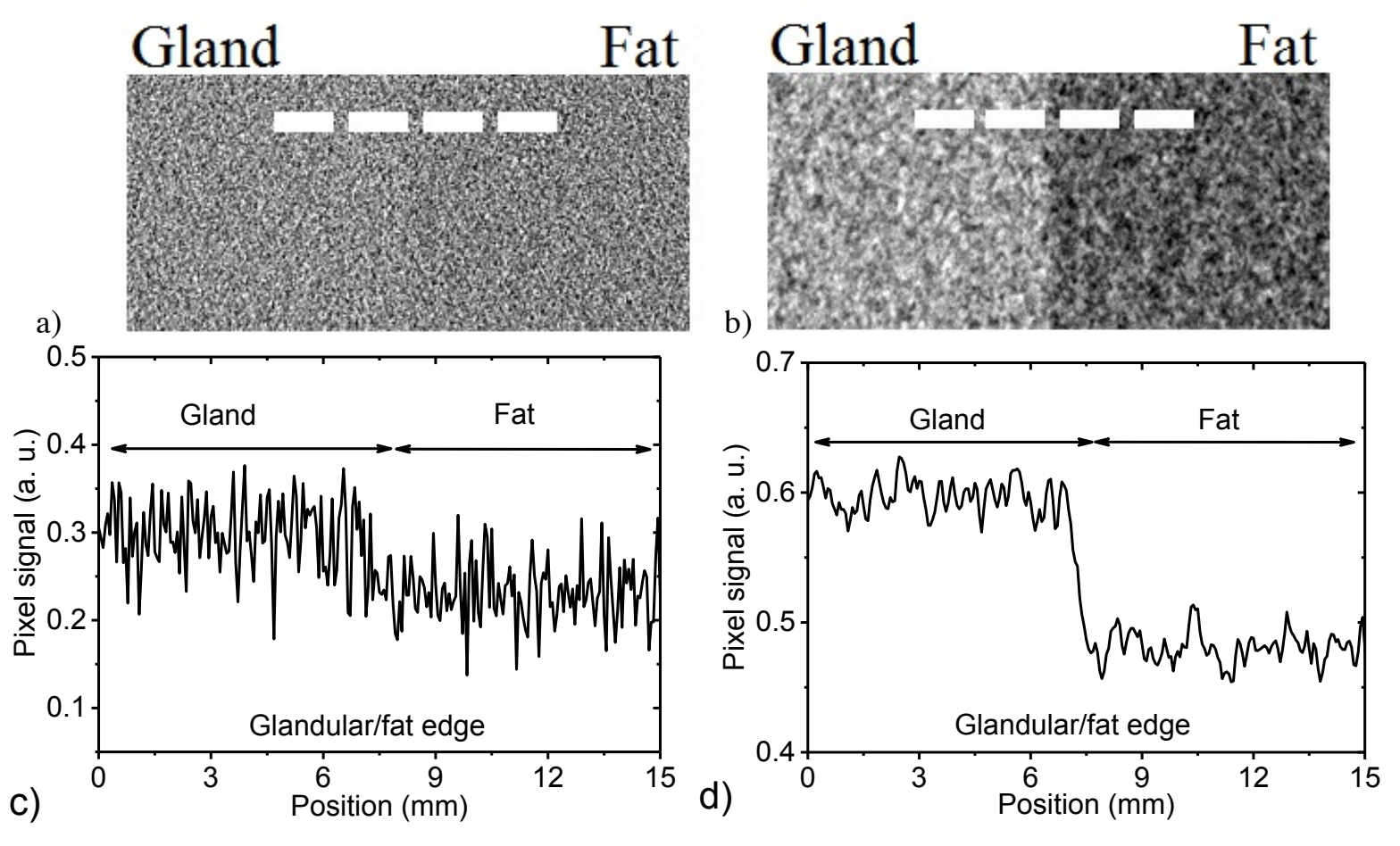

Figure 2: Glandular-fat step-wedge images (a) without phase retrieval and (b) with phase-retrieval extracted from slices with voxel size of $(60 \mu \mathrm{m})^{3}$ reconstructed via FBP algorithm. Signal intensity profiles across the dashed lines (top images) (c) without phase-retrieval and (d) with phase-retrieval averaged over 100 adjacent rows.

In principle it could be possible to reduce the noise level by applying a filter to the reconstructed image, at the cost of a reduction in spatial resolution. Figure 3 compares the FWHM as a function of the CNR for the reconstruction with phase retrieval and for that without phase retrieval, the latter one unfiltered (sigma $=0$ ) or filtered through a Gaussian filter with varying sigma. It can be observed that when the FWHM of the reconstruction without phase retrieval becomes comparable to that with phase retrieval, the corresponding CNR value is significantly lower. 


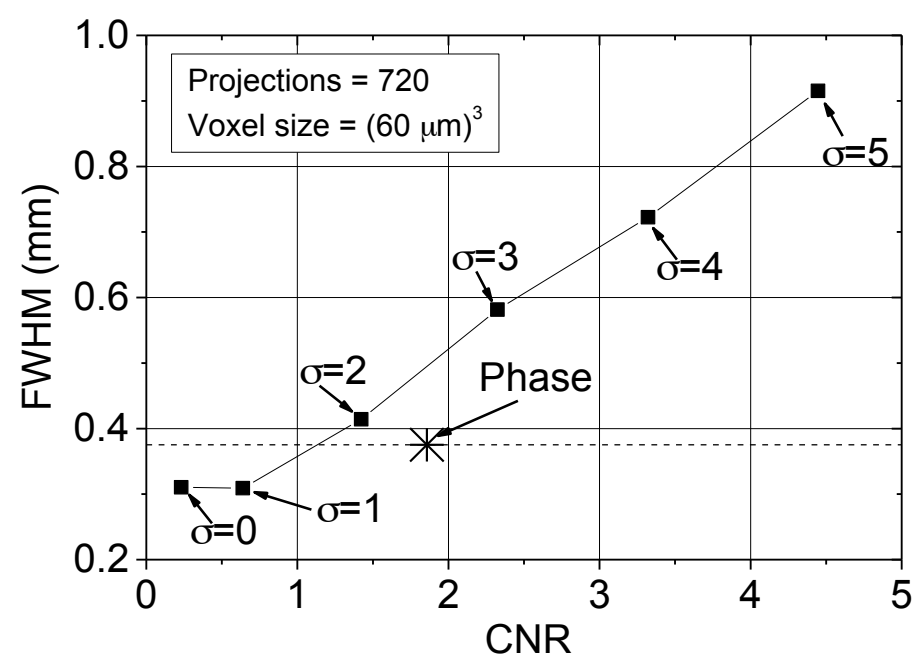

Figure 3: FWHM vs CNR with different Gaussian-filter widths applied to the reconstructed image without phase-retrieval compared to CNR and FWHM of the phase-retrieved slice (* symbol).

Tomographic data sets were acquired from breast tissue specimens in order to perform a preliminary evaluation of PhC breast CT images of potential clinical interest. In Figure 4, image reconstructions of the sample 1 are presented. Figure 4a has been obtained with the FBP algorithm by using 1200 projections and a $\mathrm{MGD}_{\mathrm{v}}$ of $23 \mathrm{mGy}$. The voxel of the reconstructed images is $(120 \mu \mathrm{m})^{3}$, as a good compromise between spatial resolution and signal-to-noise ratio. The black details inside the object are air bubbles due to the folding of the plastic bag containing the sample; edge-enhancement effects are visible at the interface airplastic foil. Figure 4b shows FBP reconstruction of 300 projections, obtained by reducing the MGD $_{v}$ (about 6 $\mathrm{mGy}$ ) of a factor 4 with respect to figure $4 \mathrm{a}$. Figure $4 \mathrm{c}$ shows the same 300 -projections data set reconstructed by means of SART $\left(\alpha_{d}=4\right.$ and $\left.\alpha_{r}=0.2\right)$. The image in figure $4 d$ was obtained by applying a phase retrieval filter prior to using the same SART algorithm used in figure 4c. Images reconstructed using SART present lower noise and a preserved spatial resolution. In contrast to figures $4 \mathrm{a}-\mathrm{c}$, in the phase retrieved image (figure 4d) the glandular tissue appears brighter than the agar gel surrounding the sample. As expected, the phase retrieved image does not show edge enhancement effects but the noise is significantly reduced. 

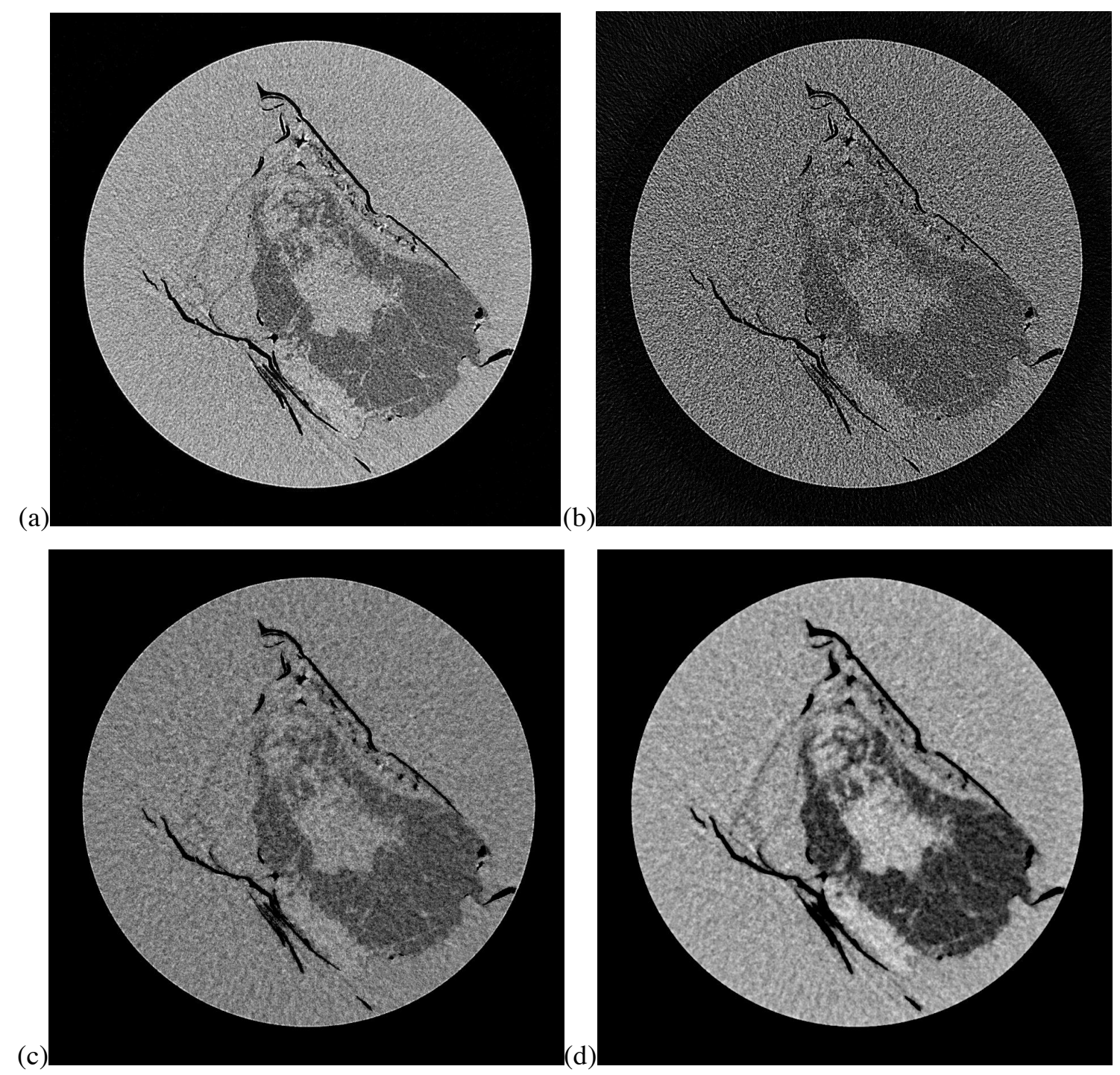

Figure 4. Sample 1 (diameter: $9.4 \mathrm{~cm}$ ).(a) FBP reconstruction of 1200 projections and (b) 300 projections, (c) SART reconstruction of 300 projections, (d) phase retrieved SART reconstruction of 300 projections.

The conventional mammography of the sample 1, before agar gel inclusion, is presented in figure 5 to show the fine parenchymal structures present in the sample. They are visible in both digital mammography and the $\mathrm{CT}$ images. However in the latter no overlapping features are present 


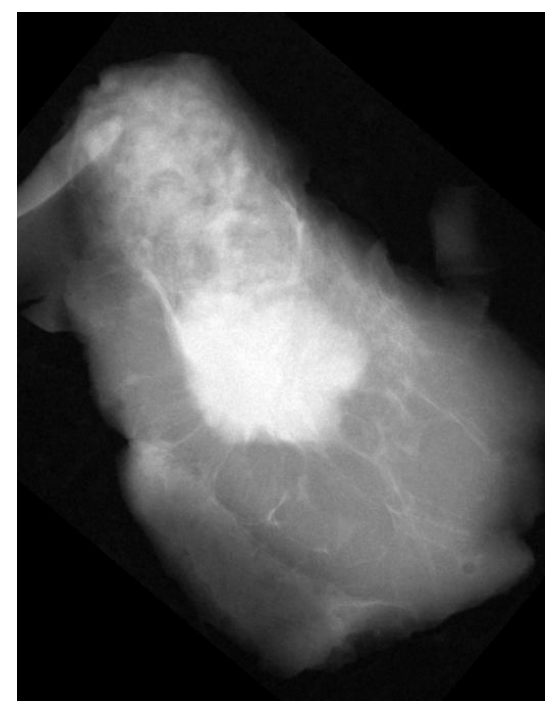

Figure 5. Digital mammography (planar image) of sample 1.

Sample 2 was studied without inclusion in the agar-gel. The $\mathrm{MGD}_{\mathrm{v}}$ was about $17 \mathrm{mGy}$ for 1200 projection over 180 degrees. According to Zhao et al (2015) in clinical breast CT, even if the voxel size is smaller than $1 \mathrm{~mm}$, the radiologist during the image evaluation adjusts the slice thickness according to the diagnostic requirements, up to about $2 \mathrm{~mm}$. In order to evaluate the potential clinical application of our low-dose images, $0.84 \mathrm{~mm}$ thick slices were obtained by adding 7 images from the sample 2 data sets. In figure 6 the results are presented for the FBP reconstruction from 1200 phase-retrieved projections (6a) and for a low dose reconstruction obtained by applying the SART technique on 300 phase retrieved projections (6b) with a dose reduction of a factor $4\left(\mathrm{MGD}_{\mathrm{v}}\right.$ is $\left.4.25 \mathrm{mGy}\right)$. Ring artefacts are visible due to the gaps between the 8 sensors of the PIXIRAD-8 detector, which were not perfectly corrected by the seaming procedure in a couple of cases. Figure $6 \mathrm{c}$ shows the planar image of the specimen obtained at a clinical mammographic unit; the nodule thickness in the sample in not constant, therefore the contrast modulation inside the cancer in the mammographic image is due to its irregular shape and not to any density inhomogeneity of the nodule. 

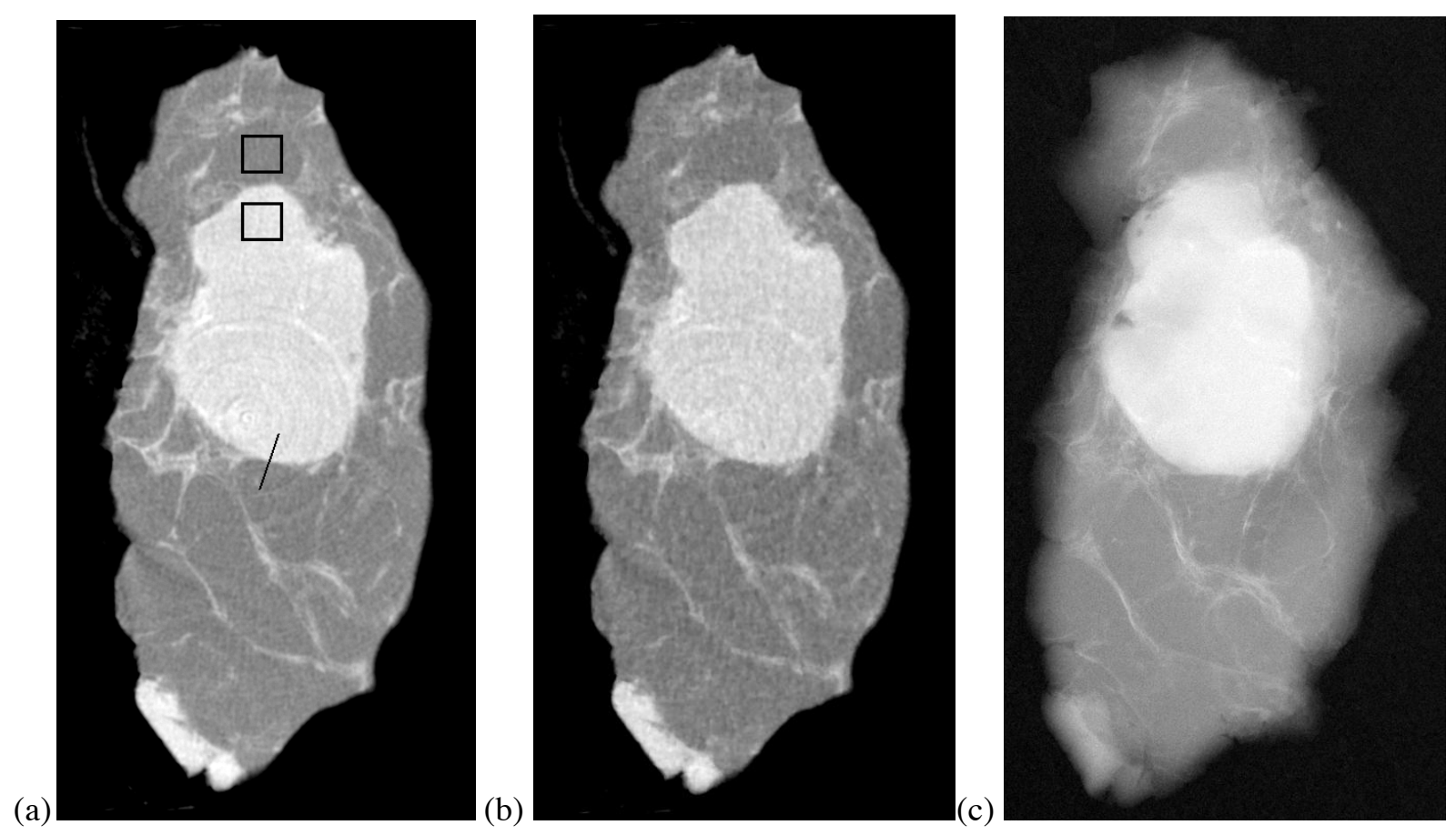

Figure 6. Images of $0.85 \mathrm{~mm}$ thick slices obtained (a) from FBP reconstruction of 1200 phase retrieved projections and (b) SART reconstruction of 300 phase retrieved projections $\left(\alpha_{\mathrm{d}}=2\right.$ and $\left.\alpha_{\mathrm{r}}=0.05\right)$. (c) Planar image obtained from the 5-mm-thick sample at a clinical mammographic unit. In (a) the ROIs for CNR and C assessment are outlined together with the line over which the profiles in figure 7 are evaluated.

In order to quantify the effect of the dose reduction and of the application of the iterative reconstruction, contrast and CNR values were calculated in both figures $6 \mathrm{a}$ and $6 \mathrm{~b}$. Moreover, the possible loss in spatial resolution, due to the reduction in projection in the SART image, has been investigated measuring the signal profiles at the boundary of the lesion in both image reconstructions. In figure $6 \mathrm{a}$, the ROIs selected for the CNR and contrast evaluation are outlined. The contrast and the CNR for the FBP reconstruction with 1200 phase retrieved projections (figure 6a) are 0.28 and 18 respectively, while, for the SART reconstruction with 300 phase retrieved projections (figure $6 \mathrm{~b}$ ), $\mathrm{C}=0.29$ and $\mathrm{CNR}=13$, indicating that the contrast is constant while a reduction of $30 \%$ of CNR is observed with a dose sparing of $75 \%$.

Figure 7 shows the signal intensity profiles of the images (evaluated across the line in figure 6a) both for the FBP reconstruction with 1200 phase retrieved projections (figure 6a) and for the SART reconstruction with 300 phase retrieved projections (figure 6b); they presented a FWHM of $420 \mu \mathrm{m}$ and resulted quite similar. The FWHM measured here is a slightly larger than the FWHM's measured from the glandular-fat stepwedge in figure 2 , due to the larger voxel (60 $\mu \mathrm{m}$ vs $120 \mu \mathrm{m}$ voxel side) and to the lack of a perfect edge in the biological sample.

These results suggest that the SART technique is a very promising candidate for the tomographic reconstruction of the SYRMA-CT images. 


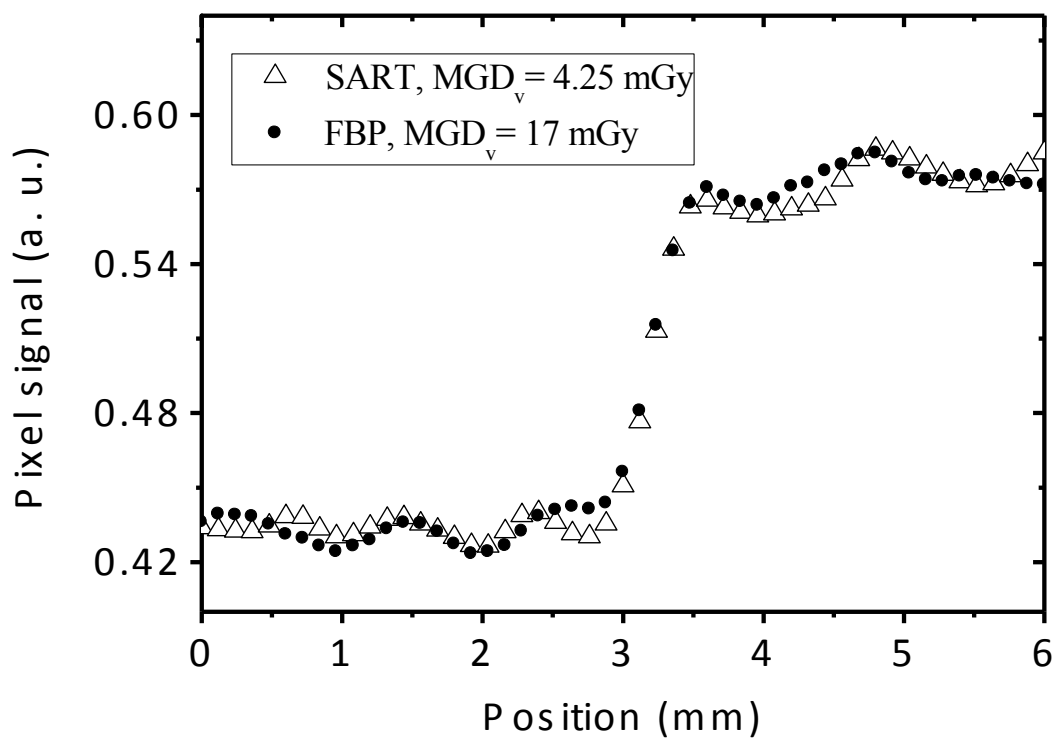

Figure 7. Profiles across the line in figure 6a, obtained both with FBP reconstruction from 1200 projections and a MGD of $17 \mathrm{mGy}$ and with SART algorithm $\left(\alpha_{\mathrm{d}}=2\right.$ and $\left.\alpha_{\mathrm{r}}=0.05\right)$ from 300 projections and $\mathrm{MGD}_{\mathrm{v}}$ of $4.25 \mathrm{mGy}$. Voxel size $=(120 \mu \mathrm{m})^{3}$.

\section{Discussion and conclusion}

In this study, monoenergetic CT scans of objects with dimensions and attenuation similar to human breast were acquired, delivering MGD in the irradiated volume $\left(\mathrm{MGD}_{\mathrm{v}}\right)$ in the range of MGD delivered in clinical breast CT (5 - $25 \mathrm{mGy}$ ) (Zhao et al 2015). Due to the spatial coherence of the SR beam and the long distance between sample and detector, the images contain additional phase information, which was exploited in the reconstruction process by applying a phase-retrieval procedure with an important improvement in the image quality. Our preliminary results show that, by applying SART iterative reconstruction to a reduced number of projections (300), images can be obtained that present the same spatial resolution and a CNR decrease by $30 \%$ with reduction by a factor 4 of the absorbed dose, if compared to images reconstructed with 1200 projections and FBP.

The first images of the SYRMA-CT collaboration reported in this paper on a breast specimen of about $9 \mathrm{~cm}$ in diameter indicate that clinical PhC breast CT with SR is technically feasible. An uncompressed breast diameter of 9-10 $\mathrm{cm}$ at chest wall corresponds to a compressed breast thickness of about $2 \mathrm{~cm}$, for which the MGD for two-view mammography is 1-2 mGy (Boone et al 2005). Hence, the minimum dose level ( $\mathrm{MDG}_{\mathrm{v}}$ about $4 \mathrm{mGy}$ ) attained in this study is only 2-4 times higher than that of two-view mammography for a 2-cm compressed breast. Noticeably, a MGD about 7 times higher than that of a two-view mammography has been reported for a $2-\mathrm{cm}$ compressed breast in a polychromatic cone-beam CT clinical prototype (Sechopoulos et al 2010). 
A general optimization of the data processing is in progress. Several reconstruction algorithms are under evaluation, including SIRT, SART, CGLS, MLEM, MR-FBP. In order to perform this optimization, a quantitative evaluation of image quality is required. This, however, is a challenging task, in particular in realdata studies in which the image may contain complex structures (Pacilè et al 2015). To take into account different aspects of image quality we are considering different parameters. Image-similarity metrics (Bian et al 2010) may be used to compare image differences in real-data studies, if a reference image is available. Visibility parameters, like the contrast-to-noise ratio (CNR) or the CNR normalized by the dose are also relevant (Gureyev et al. 2014), if the signal and the background can be clearly identified in the image. Likewise, the evaluation of spatial resolution is a significant task to compare different acquisition and reconstruction techniques and will be taken into account. The PIXIRAD pixel pitch is $60 \mu \mathrm{m}$ and the isotropic voxel size of the presented images of breast tissue samples is $(120 \mu \mathrm{m})^{3}$; further investigations are necessary in order to define the best spatial resolution for clinical application, considering that a larger voxel size could be associated to a further reduction of the delivered dose. In the meantime, the upgrading of the beamline control and safety system has been carried on in order to move from planar mammography to breast CT and the medical protocol is under development.

Other research groups reported CT images of breast samples obtained with different phase contrast techniques. Grandl et al (2014) studied a breast sample with fibroadenoma using grating-based $\mathrm{PhC}$ tomography: the results were impressive in terms of tissues discrimination, but the acquisition time of the order of many hours and the small dimension of the sample $(3 \mathrm{~cm}$ diameter $)$ indicate that the technique is not ready for clinical application. Zhao et al (2012) published a CT study of a large breast sample $(9.5 \mathrm{~cm}$ diameter) using analyser-based monochromatic imaging (also called diffraction enhanced imaging - DEI) and delivering glandular doses comparable with breast mammography (7 mGy using FBP, 2 mGy applying EST). In order to obtain such results the beam energy was increased up to $60 \mathrm{keV}$ and the images were very different from absorption-based breast CT. The clinical application of analyser-based imaging is a matter of discussion. According to Faulconer et al (2009) it is necessary to develop an atlas of breast features to help training radiologists on how to interpret the currently unfamiliar DEI images. Under the technical point of view, the mechanical stability of the analyser set-up is very demanding, and must be assured within a few micro-radians in order to guarantee for both image quality and delivered dose. At the best of our knowledge, there are no programs of clinical trials in DEI tomography.

In medical imaging clinical trials are fundamental to evaluate the possible role of a new imaging technique in the diagnostic practice. The intended aim of the clinical trials at the SYRMEP beamline is to evaluate the image quality and the delivered dose achievable in $\mathrm{PhC}$ breast imaging with $\mathrm{SR}$, keeping in mind the time and dose constraints that are typical of the clinical practice. This will give information about the PhC breast tomography and in particular about the possible contribution of the phase retrieval to the accuracy of the diagnosis. Once these findings have been clearly established, they can set the basis for the translational research in $\mathrm{PhC}$ breast imaging, trying to preserve the relevant characteristics of SR while moving to more compact sources and finally towards the radiology departments of the hospitals. 


\section{Acknowledgments}

The authors thank dr. Maura Tonutti and prof. Maria Cova for the useful discussions.

SYRMA-CT project is supported by INFN (National Scientific Committee 5 for Technological and interdisciplinary research) and Elettra-Sincrotrone Trieste SCpA. The grant from the Fondazione CR Trieste enabled a sort of offsite breast imaging centre to be set up at the SYRMEP beam line.

\section{References}

Abrami A et al. 2005 Medical applications of synchrotron radiation at the SYRMEP beamline of ELETTRA Nucl. Instrum. Meth. A 548 221-27

Agostinelli S et al. 2003 Geant4 - A Simulation toolkit Nucl. Instrum. Meth. A 506 250-303

Arfelli F et al. 1997 A linear array silicon pixel detector: Images of a mammographic test object and evaluation of delivered doses Phys. Med. Biol. 43 1565-73

Arfelli F et al. 1998 Low-dose phase contrast x-ray medical imaging Phys. Med. Biol. 43 2845-52

Åslund M, Cederström B, Lundqvist M and Danielsson M 2007 Physical characterization of a scanning photon counting digital mammography system on Si-strip detectors Med. Phys. 34 1918-25

Bellazzini R, Spandre G, Brez A, Minuti M, Pinchera M and Mozzo P 2013 Chromatic X-ray imaging with a fine pitch CdTe sensor coupled to a large area photon counting pixel ASIC J Instrum 8 C02028

Beltran M A, Paganin D M, Siu K K, Fouras A, Hooper S B, Reser D H and Kitchen M J 2011 Interfacespecific x-ray phase retrieval tomography of complex biological organs Phys. Med. Biol. 56, 735369.

Bian J, Siewerdsen J H, Han X, Sidky E Y, Prince J L, Pelizzari C A and Pan X 2010 Evaluation of sparseview reconstruction from flat-panel-detector cone-beam CT Phys. Med. Biol. 55 6575-99

Bian J, Yang K, Boone J M, Han X, Sidky E Y and Pan X 2014 Investigation of iterative image reconstruction in low-dose breast CT Phys. Med. Biol. 59 2659-85

Boone J M, Kwan A L C, Seibert J A, Sha N and Lindfors K K 2005 Technique factors and their relationship to radiation dose in pendant geometry breast CT Med. Phys. 32 3767-76

Bravin A, Coan P and Suortti P 2013 X-ray phase-contrast imaging: from pre-clinical applications towards clinics Phys. Med.Biol. 58 R1-R35

Brun E, Grandl S, Sztrókay-Gaul A, Barbone G, Mittone A, Gasilov S, Bravin A and Coan P 2014 Breast tumor segmentation in high resolution x-ray phase contrast analyzer based computed tomography Med.Phy. 41 111902-9

Brunetti E and Golosio B 2001 A new algorithm for Computer Tomographic Reconstruction from partial view projections Med. Phy. 28 462-68

Buzug T 2008 Computed Tomography: From Photon Statistics to Modern Cone-Beam CT. New York, NY, USA: Springer 
Camerani M C, Golosio B, Somogyi A, Simionovici A S, Steenari B M and Panas E 2004 X-ray fluorescence tomography of individual municipal solid waste and biomass fly ash particles Anal. Chem. 76(6) 1568-95

Castelli E et al 2011 Mammography with synchrotron radiation: first clinical experience with PhaseDetection technique Radiology 250 684-94

Cauzid J, Philippot P, Bleuet P, Simionovici A, Somogyi A and Golosio B 2007 3D imaging of vapour and liquid inclusions from the Mole Granite, Australia, using helical fluorescence tomography Spectrochim. Acta B 62 799-806

Chen R C, Rigon L and Longo R 2013 Comparison of single distance phase retrieval algorithms by considering different object composition and the effect of statistical and structural noise Optics Express 21 7384-99

Chmeissani M et al. 2004 First Experimental Tests with a CdTe Photon Counting Pixel Detector Hybridized with a Medipix2 Readout Chip. IEEE Trans. Nucl. Sci. 51, 2379-85

Cloetens P, Ludwig W, Baruchel J, Van Dyck D, Van Landuyt J, Guigay JP, and Schlenker M 1999 Holotomography: Quantitative phase tomography with micrometer resolution using hard synchrotron radiation x rays Appl. Phys. Lett. 75 2912-2914

Coan P, Bravin A and Tromba G 2013 Phase-contrast x-ray imaging of the breast: recent developments towards clinics J. Phys. D: Appl. Phys 46 e494007

Dance D R, Young K C and van Engen R E 2009 Further factors for the estimation of mean glandular dose using the United Kingdom, European and IAEA dosimetry protocols Phys. Med. Biol. 56 4361-72

Dempster A P, Laird N M, and Rubin D B 1977 Maximum likelihood from incomplete data via the em algorithm Journal of the Royal Statistical Society, Series B 39 1-38

Faulconer L et al. 2009 Radiologist evaluation of an X-ray tube-based diffraction-enhanced imaging prototype using full-thickness breast specimens Acad. Radiol. 16 1329-37

Fedon C, Longo F, Mettivier G, Longo R 2015 GEANT4 for breast dosimetry: parameters optimization study Phys. Med.Biol. 60 N311-N323

Grandl S et al. 2014 Visualizing typical features of breast fibroadenomas using Phase-Contrast CT: an ExVivo study PlosOne 9(5) e97101

Golosio B, Brunetti A and Cesareo 2004 Algorithmic techniques for quantitative Compton tomography Nucl. Instrum. Methods B 213 108-11

Gordon R, Bender R, Herman GT 1970 Algebraic Reconstruction Techniques (ART) for three-dimensional electron microscopy and X-ray photography Journal of Theoretical Biology 29 471-481

Gureyev T E et al. 2014 Investigation Of Imaging Quality of Synchrotron-Based Phase-Contrast Mammographic Tomography J. Phys. D: Appl. Phys 47365401

Huang S Y, Boone J M, Yang K, Packard N J, McKenney S E, Prionas N D, Lindfors K K and Yaffe M Y 2011 The characterization of breast anatomical metrics using dedicated breast CT Med. Phys. 38 2180-91 
Kak A C and Slaney M 1988 Principles of Computerized Tomographic Imaging (IEEE Press)

Kalender W A, Beister M, Boone J M, Kolditz D, Vollmar S V and Weigel M C C 2012 High-resolution spiral CT of the breast at very low dose: concept and feasibility consideration Eur. Radiol. 22 1-8

Longo R et al. 2003 Morphological breast imaging: tomography and digital mammography with synchrotron radiation Nucl. Instrum. and Meth. A 497 9-13

Longo R et al. 2014 Clinical study in phase-contrast mammography: image-quality analysis Phil. Trans. R. Soc. 37220130025

Lopez F C, Rigon L, Fardin L, Arfelli F, Bergamaschi A, Dreossi D, Longo M, Schmitt B, Vallazza E and Longo R 2014 Comparator threshold settings and the effective pixel width of the PICASSO detector JINST 9 C05056

Makeev A and Glick S J 2013 Investigation of statistical iterative reconstruction for dedicated breast CT Med.Phy. 40081904

Mettivier G, Fedon C, Di Lillo F, Longo R, Sarno A, Tromba G and Russo P 2015 Glandular dose in breast computed tomography with synchrotron radiation Phys. Med.Biol. (accepted for publication)

Miao J, Förster F and Levi O 2005 Equally Sloped Tomography with Oversampling Reconstruction Phys. Rev.B. 72052103

Mittone A, Bravin A and Coan P 2014 Radiation dose in breast CT imaging with monochromatic x-rays: Simulation study of the influence of energy, composition and thickness Phys Med Biol 59 2199-2217

Nesterets Y I and Gureyev T E 2014 Noise propagation in x-ray phase-contrast imaging and computed tomography J. Phys. D 47, 105402.

Nesterets Y I et al. 2016 A feasibility study of X-ray phase-contrast mammographic tomography at the Imaging and Medical beamline of the Australian Synchrotron J. Synchrotron Rad. 23, (in press)

O'Connell A M, Karellas A and Vedantham S 2014 The potential role of dedicated 3D breast CT as a diagnostic tool: review and early clinical examples Breast Journal 20 592-605

Pacilè S et al. $2015 \mathrm{X}$-ray phase-contrast imaging: from pre-clinical applications towards clinics Biomed. Opt. Express 6, 3099

Paganin D, Mayo S C, Gureyev T E, Miller P R and Wilkins S W 2002 Simultaneous phase and amplitude extraction from a single defocused image of a homogeneous object J. Micros. 206 33-40

Pani S et al. 2004 Breast tomography with synchrotron radiation: preliminary results Phys. Med. Biol. 49 1739-54

Pelt D M and Batenburg K J 2014 Improving filtered backprojection reconstruction by data-dependent filtering IEEE Transactions on Image Processing 23 4750-62

Prionas N D, Lindfors K K, Ray S, Huang S Y, Beckett L A, Monsky W L and Boone J M 2010 Contrastenhanced Dedicated Breast CT: Initial Clinical Experience Radiology 256 714-23

Quai E et al. 2012 First application of computed radiology to mammography with synchrotron radiation Radiologia Medica 118 89-100

Sarno A, Mettivier G and Russo P 2015 Dedicated breast computed tomography Med. Phys. 42 2786-2804 
Sechopoulos I, Feng S S J and D'Orsi C J 2010 Dosimetric characterization of a dedicated breast computed tomography clinical prototype Med. Phys. 37 4110-20

Sechopoulos I 2013 A review of breast tomosynthesis. Part I. The image acquisition process Med. Phys. 40 014301-12

Sechopoulos I 2013 A review of breast tomosynthesis. Part II. Image reconstruction, processing and analysis, and advanced applications Med. Phys. 40 014302-17

Taguchi K and Iwanczyk J S 2013 Vision 20/20: Single photon counting x-ray detectors in medical imaging Med.Phys. 40 100901-19

Tanaka $\mathrm{T}$ et al. 2005 The first trial of phase contrast imaging for digital full-field mammography using a practical molybdenum x-ray tube Invest. Radiol. 40 385-96

Van der Sluis A and Van der Horst HA 1990 SIRT and CG-type methods for the iterative solution of sparse linear least-squares problems Linear Algebra Appl. 130 257-302

Vincenzi A, De Ruvo P L, Delogu P, Bellazzini R, Brez A, Minuti M, Pinchera M, Spandre G 2015 Energy characterization of Pixirad-1 photon counting detector system J. Instrum. 10 C04010

Vedantham S, Shi L, Karellas A , O'Connell A M and Conover D L 2013 Personalized estimates of radiation dose from dedicated breast $\mathrm{CT}$ in a diagnostic population and comparison with diagnostic mammography Phys. Med. Biol. 58 7921-7936

Weigel S, Berkemeyer S, Girnus R, Sommer A, Lenzen H and Heindel W 2014 Digital Mammography screening with Photon-counting technique: Can a high diagnostic performance be realized at low mean glandular dose? Radiology 271 345-55

Zhao B, Zhang X, Cai W, Conover D and Ning R 2015 Cone beam breast CT with multiplanar and three dimensional visualization in differentiating breast masses compared with mammography Eur. $J$. Radiol. 84 48-53

Zhao $\mathrm{Y}$ et al. 2012 High resolution, low dose phase contrast $\mathrm{x}$-ray tomography for 3D diagnosis of human breast cancers Proc. Natl. Acad. Sci. USA 109 18290-94 